Results A minimum wear time of 11 hours ( $\geq 1$ valid day) allowed $80 \%$ of the sample to be retained. All minimum wear time thresholds produced an ICC $\geq 0.80$, resulting in 1 day of wear required to produce representative inpatient PA. Mornings and afternoons were more active than evenings and overnight $(32.1 \%$ and $32.0 \%$ vs. $25.2 \%$ and $10.7 \%$ of steps/day, respectively, $\mathrm{p}<0.001$ ) (figure 1 ). No changes in PA were observed during the hospital stay; ranging 585-707 steps/day and $72-83 \mathrm{~min} /$ day of walking. After controlling for wear time, patients admitted for 2-3 days took more steps on average than patients staying $7-14$ days $(997 \pm 125$ vs. $597 \pm 91$, $\mathrm{p}=0.036$ ).

Conclusions One full day ( 24 hours) of monitoring is required at the individual-level to obtain representative inpatient PA. A minimum wear time criteria of $\geq 11$ waking hours is recommended for sample-level data. Wear time and LOHS should be accounted for in analyses.

\section{S83 NEURAL RESPIRATORY DRIVE, RESPIRATORY MECHANICS AND BREATHLESSNESS IN HIGHLY ACTIVE OLDER ADULTS}

${ }^{1}$ AS Shaar, ${ }^{1} B$ Best, ${ }^{2} \mathrm{M}$ Choudhury, ${ }^{1} \mathrm{Z}$ Berger, ${ }^{1} \mathrm{SDR}$ Harridge, ${ }^{1} \mathrm{NR}$ Lazarus, ${ }^{1} \mathrm{CJ}$ Jolley. ${ }^{1}$ King's College London, London, UK; ${ }^{2}$ King's College Hospital NHS Foundation Trust, London, UK

\subsection{6/thoraxjnl-2017-210983.89}

Introduction and Objectives Ageing is typically associated with progressive deleterious changes in respiratory mechanics which increase the work of breathing and limit ventilatory reserve. The impact on exercise capacity and exertional breathlessness in healthy ageing remains incompletely understood, in part due to a failure of previous research to control for negative effects of physical inactivity. This study aimed to compare neural respiratory drive (NRD), respiratory mechanics and breathlessness between highly active older adults (AOA) and recreationally active younger adults (YA). We hypothesised that NRD, quantified as diaphragm electromyogram activity (EMGdi) as a percentage of volitional maximum (EMGdi\% max), would be higher in AOA than in YA and that this would be associated with increased breathlessness intensity during exercise.

Methods 12 YA (mean (+/-SD) age 26.4+/-4.7 years) and 12 AOA (cyclists, 59.0+/-10.1 years), all male, underwent incremental cycle ergometry to their symptom-limited maximum. EMGdi was recorded continuously using an oesophageal multipair electrode catheter and quantified as EMGdi\%max. Breathlessness intensity was quantified each minute and at endexercise using the modified Borg scale.

Results Absolute $\mathrm{FEV}_{1}, \mathrm{FVC}, \mathrm{FEV}_{1} \% \mathrm{FVC}$ and IC tended to lower values in AOA than in YA (Table 1), without significant differences in baseline EMGdi\%max (AOA 10.6+/-5.8\%max; YA 7.6+/-3.7\%max, $p=0.15)$. End-exercise EMGdi\%max was significantly higher in AOA than in YA (AOA 61.8+/-13.7\% $\max$; YA: $51.9+/-9.1 \% \max \mathrm{p}=0.049)$, with a trend towards higher end-exercise tidal volume $\left(\mathrm{V}_{\mathrm{T}}\right)$ relative to IC (AOA: $\mathrm{V}_{\mathrm{T}} \% \mathrm{IC}=83.1+/-16.7 \% ; \quad \mathrm{YA}: \quad \mathrm{V}_{\mathrm{T}} \% \mathrm{IC}=73.2+/-17.1 \%$, $\mathrm{p}=0.17)$. There were no significant end-exercise differences in $\mathrm{V}_{\mathrm{T}}$ (AOA 2.9+/-0.8 L; YA 2.8+/-0.6 L, p=0.85), minute ventilation $\left(\mathrm{V}_{\mathrm{E}}\right) \quad(\mathrm{AOA} 116.7+/-35.8 \mathrm{~L} / \mathrm{min}$; YA: $110.4+/-29.3 \mathrm{~L} /$ $\min , \mathrm{p}=0.64$ ) or mBorg breathlessness intensity (median (IQR) AOA 5 (3.25-7.75); YA 5 (5-9), $\mathrm{p}=0.5)$.
Conclusions Highly active older adults achieved a similar endexercise $V_{E}$ to the younger adults despite age-related respiratory constraints. This required higher levels of NRD, which contrary to our hypothesis, was not perceived as increased breathlessness intensity. The contribution of ageing and/or regular physical activity to this apparent blunted perception of breathlessness requires further study.

Abstract S83 Table 1 Demographics, anthropometrics and lung function data for active older adults (AOA) and younger adults (YA). $\mathrm{BMI}$, body mass index; $\mathrm{FEV}_{1}$, forced expiratory volume in $1 \mathrm{~s}$; FVC, forced vital capacity; TLC, total lung capacity; RV, residual volume; IC, inspiratory capacity. Values presented are mean \pm SD. * Indicates statistically significant difference between groups, $\mathrm{p}<0.05$

\begin{tabular}{llll}
\hline & $\begin{array}{l}\text { Younger } \\
\text { adults }\end{array}$ & $\begin{array}{l}\text { Active Older } \\
\text { Adults }\end{array}$ & p-value \\
\hline Age (yrs) & $26.4 \pm 4.7$ & $59.0 \pm 10.1$ & $<0.0001^{*}$ \\
BMI (kg/m ${ }^{2}$ ) & $24.5 \pm 3.5$ & $25.7 \pm 2.4$ & 0.35 \\
FEV 1 (L) & $4.70 \pm 0.80$ & $3.82 \pm 0.86$ & $0.023^{*}$ \\
FEV $\%$ pred. (\%) & $105.0 \pm 17.9$ & $109.1 \pm 12.8$ & 0.53 \\
FVC (L) & $5.88 \pm 0.95$ & $5.23 \pm 1.20$ & 0.16 \\
FVC\% pred. (\%) & $105.6 \pm 15.9$ & $112.1 \pm 16.8$ & 0.11 \\
FEV $\%$ FVC (\%) & $79.5 \pm 5.0$ & $73.1 \pm 6.1$ & $0.01^{*}$ \\
IC (L) & $3.80 \pm 0.47$ & $3.47 \pm 0.86$ & 0.26 \\
IC pred. (\%) & $89.4 \pm 17.8$ & $97.6 \pm 21.6$ & 0.40 \\
TLC (L) & $7.68 \pm 1.12$ & $7.76 \pm 1.78$ & 0.91 \\
TLC\% pred. (\%) & $105.0 \pm 13.6$ & $106.4 \pm 17.2$ & 0.84 \\
RV (L) & $1.95 \pm 0.26$ & $2.41 \pm 0.70$ & 0.09 \\
RV\% pred. (\%) & $110.1 \pm 17.9$ & $97.6 \pm 25.0$ & 0.24 \\
RV\% TLC (\%) & $26.0 \pm 4.2$ & $31.3 \pm 6.0$ & $0.043^{*}$ \\
\hline
\end{tabular}

\section{S84 DO PATIENTS GAIN AS MUCH KNOWLEDGE AROUND THEIR CONDITION FROM A WEB-BASED PULMONARY REHABILITATION PROGRAMME?}

${ }^{1} \mathrm{E}$ Chaplin, ${ }^{1} \mathrm{~S}$ Hewitt, ${ }^{1,2} \mathrm{~S}$ Singh. ${ }^{1}$ Centre for Exercise and Rehabilitation Science, Pulmonary Rehabilitation Department, Glenfield Hospital, University Hospitals of Leicester NHS Trust, Leicester, UK; ${ }^{2}$ School of Sport, Exercise and Health Sciences, Loughborough University, Loughborough, UK

\subsection{6/thoraxjnl-2017-210983.90}

Introduction The educational components of Pulmonary Rehabilitation (PR) are fundamental to the format and success of the programme. ${ }^{1}$ All patients enrolled onto a PR programme should have access to a structured education programme. However the ideal mode of delivery is unknown and delivery can be varied. It has previously been demonstrated that a web-based programme is feasible. ${ }^{2}$ However the gains in knowledge have not previously been reported for a digital programme compared to face-to-face contact. The Bristol COPD knowledge questionnaire (BCKQ) is a 65 item selfcompleted questionnaire often used to assess the effectiveness of education delivered.

Methods A single blinded, randomised, controlled feasibility trial comparing a web-based PR programme with a conventional PR programme for 7 weeks was conducted. Patients completed a BCKQ at their initial assessment or online when registering and again at their discharge appointment following completion of the PR programme or online at the end of the web programme. 